\title{
Dynamic range extension method for microwave power meters
}

\author{
Alexander Cherepanov ${ }^{1, *}$, Alexander Dobrikov ${ }^{1}$, and Andrew Zagorodny ${ }^{2}$ \\ ${ }^{1}$ Micran Research and Production Company, 634041, 51d Kirova Avenue, Tomsk, Russia \\ ${ }^{2}$ Tomsk State University of Control Systems and Radio Electronics, 634050, 40, prospect Lenina, \\ Tomsk, Russia
}

\begin{abstract}
The paper is devoted to microwave power meters, detector blocks of which can be located directly on the main PC board. The block diagram with dynamic range extension method was shown. Portable PLS series power meters with frequency range from $50 \mathrm{MHz}$ to $6 \mathrm{GHz}$ produced by Micran Company is presented. Galvanic isolation unit is proposed.
\end{abstract}

\section{Introduction}

Development of modern technology requires improvement of the technology for the provision of information and telecommunication services (satellite communications, television, radio navigation, etc.) using electromagnetic radiation in the microwave range. Power is the main energy parameter for microwave signals [1]. Telecommunication development leads to a complication of the RF signals, the requirements for characteristics of power meters, such as frequency and dynamic ranges, accuracy, and the capability to measure modulated signals, including different digital modulation types and pulse modulation.

It's known few main power sensors types: bolometer, calorimeter, diode detector [2]. Bolometers and calorimeters are characterized by such disadvantages as high inertness, low level of sensitivity, low measurement speed. Advantages of diode detectors: speed, high sensitivity and small size.

In this paper, we consider one of the options for the implementation of microwave power meters with a dynamic range of $70 \mathrm{~dB}$ developed and manufactured by Micran research and production company (Tomsk, Russia) [3].

\section{Microwave power meter dynamic range for modulated signals}

For power measurements typically use detector diodes operating in their square law region, or that region of the detection curve where detected voltage is proportional to RF input power. For a typical packaged diode, the square-law detection region exists from the noise level up to approximately $-20 \mathrm{dBm}$. The transition region ranges from approximately -20 to $0 \mathrm{dBm}$ input power, while the linear detection region extends above approximately 0

* Corresponding author: cherepanov.av@micran.ru 
$\mathrm{dBm}$ [4]. Measurement of modulated signal power more than $-20 \mathrm{dBm}$ will be performed with an error. One of the methods to expand the area of square-law detection of diode power meters is to expand the dynamic range by using two or more detection circuits [5]. The block diagram is shown in Figure 1.

In the two-path detector, a signal is supplied to each detector section with different attenuation, and both detectors work on square-law detection region. This method allows you to increase the dynamic range up to $90 \mathrm{~dB}$ (Figure 2).

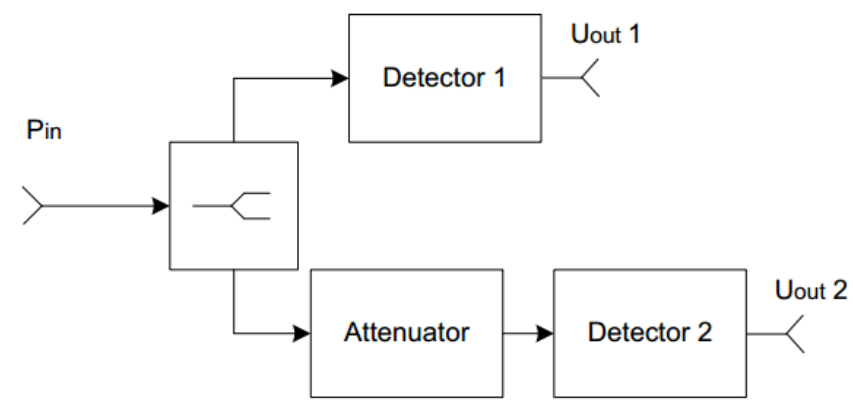

Fig. 1. Two-path detector block diagram.

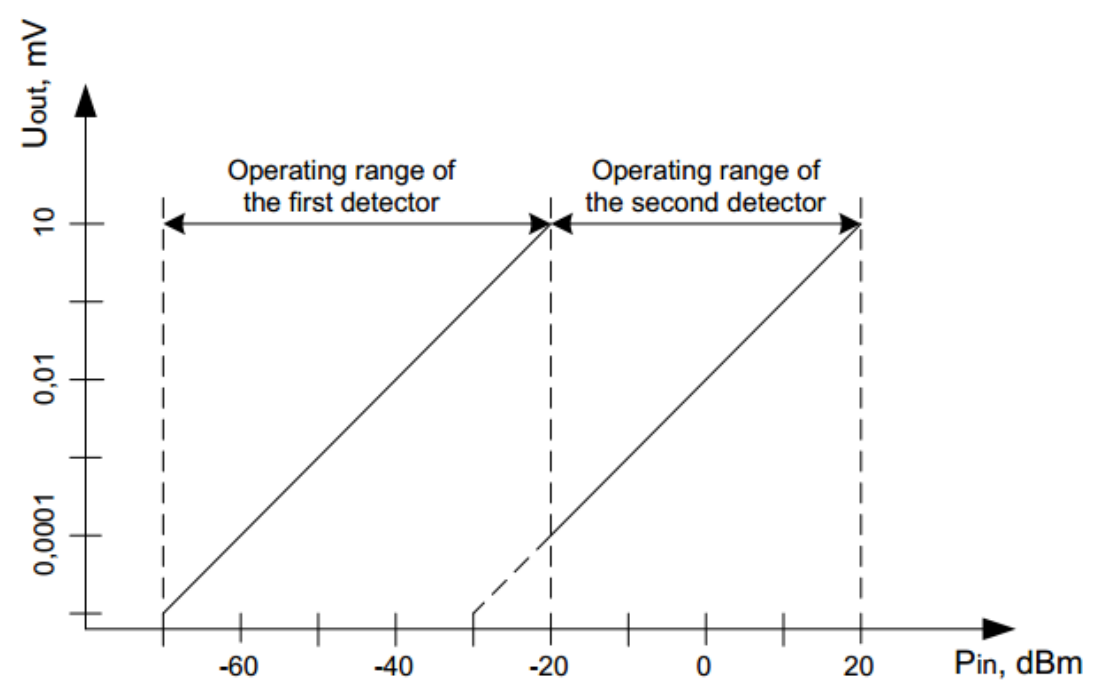

Fig. 2. Output voltages vs input power for two-path detector.

LadyBug Technologies presents its portable power meter solution without the need for zeroing and calibration. The circuit uses two detector sections to expand the dynamic range $[6,7]$. The block diagram is shown in Figure 3.

After a power splitter, in conjunction with an NBB-312 wideband amplifier on one detection path and an attenuator on the other, a signal of different levels goes to two AD8318 logarithmic detectors, then it is digitized using the AD7655 analog-to-digital converter. The measurement results via the USB interface implemented on the CY7C68 controller are transmitted to a computer. The disadvantage of this solution is the use of logarithmic detectors, which do not allow measuring digital modulated signals with good accuracy. 


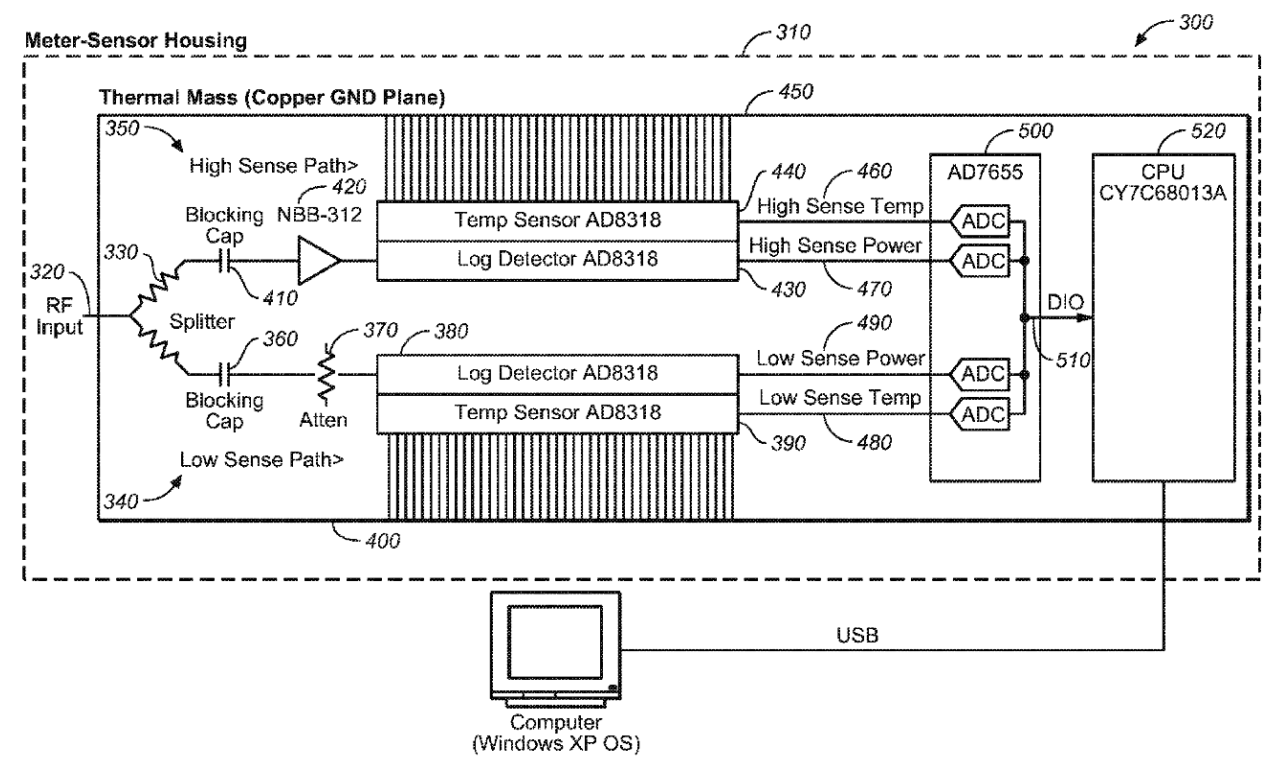

Fig. 3. Block diagram of a LadyBug Technologies power meter.

The authors propose portable power meter with power and control via USB, with a dynamic range from $-50 \mathrm{dBm}$ to $20 \mathrm{dBm}$ and a frequency range from $50 \mathrm{MHz}$ to $6 \mathrm{GHz}$. The block diagram is shown in Figure 4.

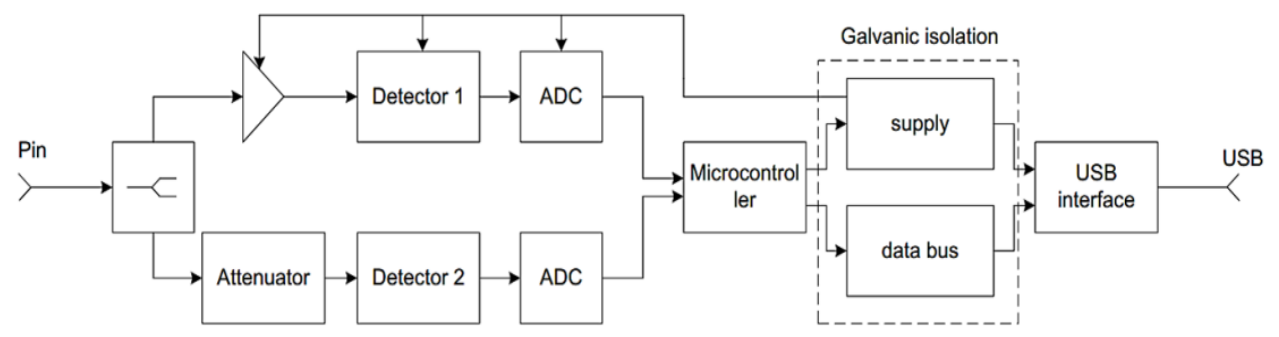

Fig. 4. PLS06 block diagram.

The proposed solution differs from its foreign counterpart:

- Using an asymmetric power divider, which allows to reduce the lower limit of detection and simplify the requirements for the attenuator;

- The use of microwave signal power detectors, which respond to the RMS value of the input signal;

- The presence of galvanic isolation in order to avoid the influence of the potential difference.

\section{Asymmetric power divider calculation}

Power dividers based on "Star" or "Triangle" circuits and power splitters provide $6 \mathrm{~dB}$ loss to each output. The asymmetric divider makes it possible to split the signal with $3 \mathrm{~dB}$ loss to the second port and about $16 \mathrm{~dB}$ to the third port. This allows to reduce the lower limit of detection power level and simplify the requirements for the attenuator for the second detection path. A simplified divider circuit is shown in Figure 5. 


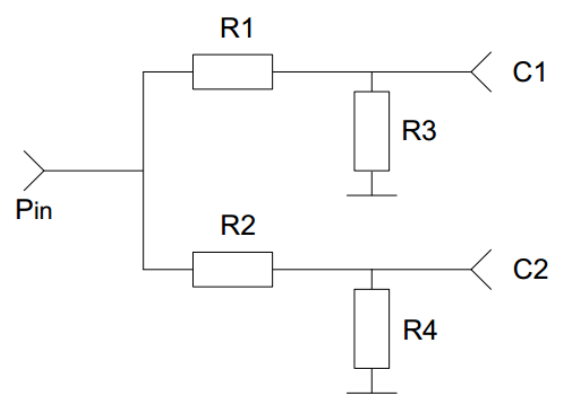

Fig. 5. Resistive power divider circuit.

The dividers input and outputs impedances must be matched to $50 \mathrm{Ohm}$. Resistors values R1- R4 are calculated based on ports matching and transmissions values to output ports.

For the scheme shown in Figure 5, formulas are compiled for calculating the transmission coefficients $\mathrm{T} 1$ and $\mathrm{T} 2$ :

$$
\begin{aligned}
& T 1=\frac{\frac{R_{3} \cdot R_{H}}{R_{3}+R_{H}}}{R_{1}+\frac{R_{3} \cdot R_{H}}{R_{2}+R_{H}}}, \\
& T 2=\frac{\frac{R_{4} \cdot R_{H}}{R_{4}+R_{H}}}{R_{2}+\frac{R_{4} \cdot R_{H}}{R_{4}+R_{H}}},
\end{aligned}
$$

where RL is the equivalent load resistance at the shoulder output.

The calculation of the equivalent input resistance of the divider and each arm is presented in the formulas:

$$
\begin{aligned}
& R_{E}=\frac{\left(R_{1}+\frac{R_{3} \cdot R_{L}}{R_{3}+R_{L}}\right) \cdot\left(R_{2}+\frac{R_{4} \cdot R_{L}}{R_{4}+R_{L}}\right)}{R_{1}+\frac{R_{3} \cdot R_{L}}{R_{3}+R_{L}}+R_{2}+\frac{R_{4} \cdot R_{L}}{R_{4}+R_{L}}}, \\
& R_{C 1}=\frac{\left(\frac{R_{E} \cdot\left(\frac{R_{4} \cdot R_{L}}{R_{4}+R_{L}}+R_{2}\right)}{R_{E}+\frac{R_{4} \cdot R_{L}}{R_{4}+R_{L}}+R_{2}}+R_{1}\right) \cdot R_{3}}{\frac{R_{E} \cdot\left(\frac{R_{4} \cdot R_{L}}{R_{4}+R_{L}}+R_{2}\right)}{R_{E}+\frac{R_{4} \cdot R_{L}}{R_{4}+R_{L}}+R_{2}}+R_{1}+R_{3}}, \\
& R_{C 2}=\frac{\left(\frac{R_{E} \cdot\left(\frac{R_{3} \cdot R_{L}}{R_{3}+R_{L}}+R_{1}\right)}{R_{E}+\frac{R_{3} \cdot R_{L}}{R_{3}+R_{L}}+R_{1}}+R_{2}\right) \cdot R_{4}}{\frac{R_{E} \cdot\left(\frac{R_{3} \cdot R_{L}}{R_{3}+R_{L}}+R_{1}\right)}{R_{E}+\frac{R_{3} \cdot R_{L}}{R_{3}+R_{L}}+R_{1}}+R_{2}+R_{4}},
\end{aligned}
$$

where $\mathrm{RC} 1$ and $\mathrm{RC} 2$ are the input resistance of the arms $\mathrm{C} 1$ and $\mathrm{C} 2$, respectively, RIN is the equivalent input resistance of the power divider.

The value of the equivalent resistance of the input of the divider RE and connected loads $\mathrm{RL}$ taken $50 \mathrm{Ohm}$. The resulting resistance values are $\mathrm{R} 1=29.6 \mathrm{Ohms}, \mathrm{R} 2=182.7$ Ohms, R3 = 153.9 Ohms and R4 =68.8 Ohms [8]. 


\section{RMS power detector}

Using RMS power detectors, in contrast to logarithmic detectors, allows measurements of modulated signals (QAM, QPSK, BPSK, OFDM) in modern communication systems. Wattmeters can also be used to measure the pulse and average power of pulse-modulated signals with a duration of more than $20 \mu \mathrm{s}$.

The power meter circuit uses RMS detectors ADL5906 with a frequency range from 10 $\mathrm{MHz}$ to $10 \mathrm{GHz}$ and a dynamic range up to $65 \mathrm{~dB}$ [9]. Compared with analogs, this detector provides the wider range of measured powers in the frequency range up to $10 \mathrm{GHz}$.

The main disadvantage of this application is that increasing frequency (from $6 \mathrm{GHz}$ to $10 \mathrm{GHz}$ ) reduces the dynamic range.

The functional diagram of the detector is shown in Figure 6.

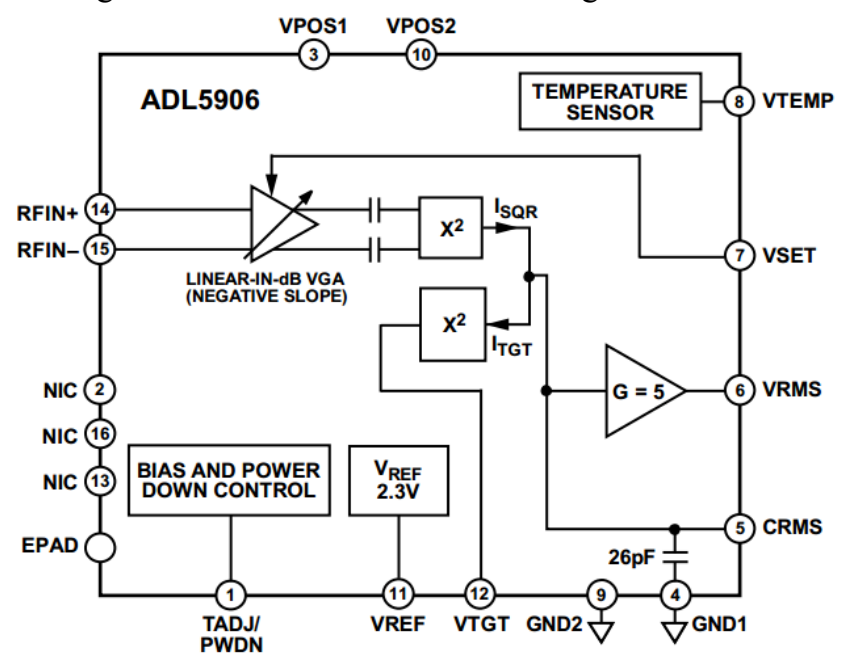

Fig. 6. ADL5906 functional diagram.

The principle of operation is based on a diode detector with bias circuits and gain control circuit of a broadband amplifier to ensure the operation in square-law region of the detector.

It means that ADL5906 power detector can detects power of modulated signals. Also IC contains a temperature sensor, for output signal swing correction.

\section{Modulated signal measurement}

One of the most important features of diode power meters is the capability to measure power of modulated signals. The possibility of such measurements is the use square-law region of the detector diode. In the presented PLS portable wattmeter, the ADL5906 detectors allow measuring pulse-modulated signals as well as digitally modulated signals.

The envelope of the modulated signal in the measurement result window of PLS-client software is shown in Figure 7.

Comparison of signal measurements with digital modulation of power meters PLS06 with analogues of foreign companies E4413A (Agilent Technologies) and the power standard NRPC (Rohde \& Schwarz) based on a thermocouple converter is carried out. The thermal sensor is optimal for comparison, since, regardless of modulation, it only responds to energy.

The digital modulated signal measurements deviation with NRPC and PLS06 power meters is shown in Figure 8, with E4413A and PLS06 power meters is shown in Figures 9. 


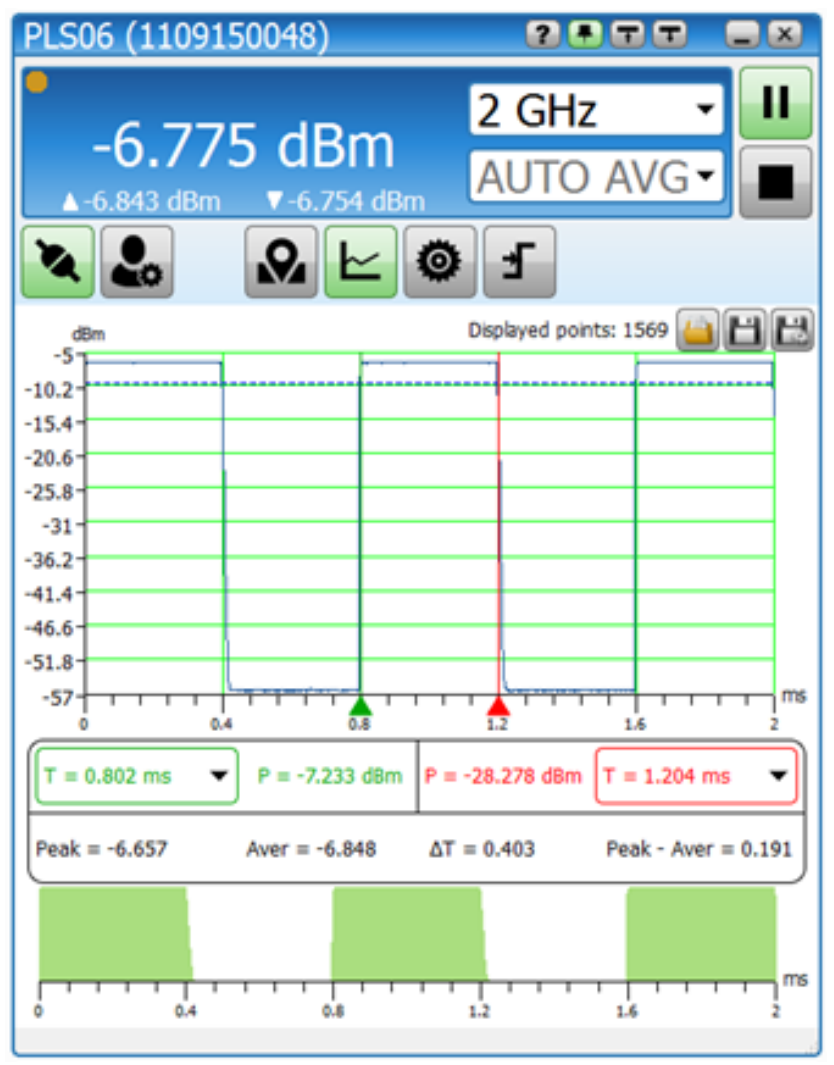

Fig. 7. The envelope of the modulated signal in PLS-client software.

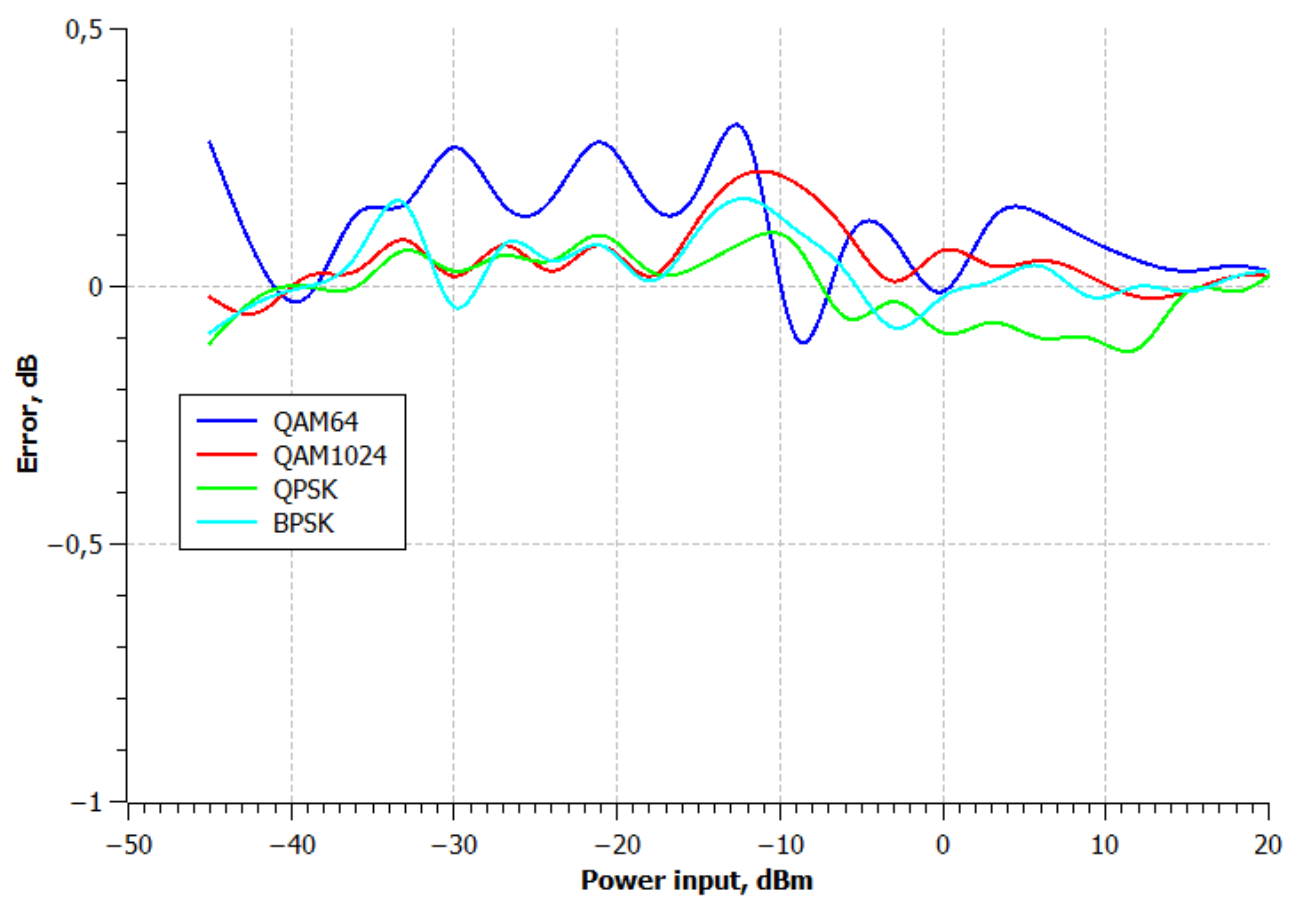

Fig. 8. The digital modulated signal measurements deviation error with NRPC and PLS06. 
Figure 8 shows the deviation of the power measurement of signals with digital modulation of QAM64, QAM1024, QPSK wattmeters NRPC and PLS06. According to the graph, it can be said that power meters throughout the entire range of input power behave identically, the measurement deviation does not exceed $0.3 \mathrm{~dB}$.

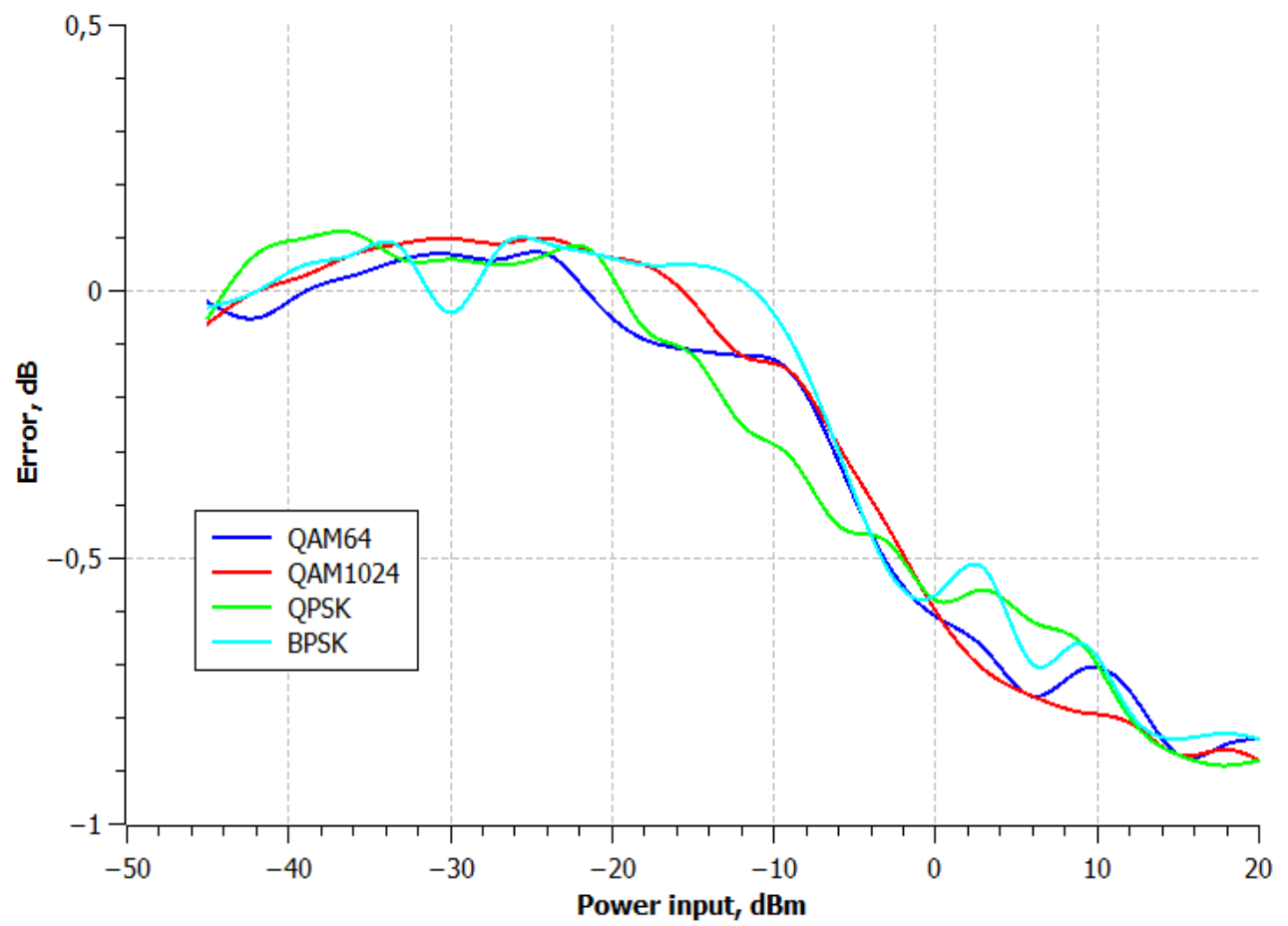

Fig. 9. The digital modulated signal measurements deviation error with NRPC and E4413A.

Figure 9 shows the deviation of the power measurement of signals with digital modulation of QAM64, QAM1024, QPSK NRPC and E4413A wattmeters. The graph shows that with an input power of minus $20 \mathrm{dBm}$ and higher, the measurement error increases.

From all of the above, it can be concluded that the PLS06 portable wattmeter is not inferior to the NRPC wattmeter in the accuracy of measuring digitally modulated signals, and even surpasses the E4413A power meter.

\section{Galvanic isolation}

In situations where two or more electric circuits must communicate, for example a few computers in wire network or computer and measurement units, their grounds may be at different potentials. When two or more units have different ground potentials there are current flows between them. Moreover, if the difference in ground potentials is sufficiently large, the resulting ground-loop current can pose a safety issue for equipment and personal.

Effective method of breaking ground loops (between units) for preventing unwanted current from flowing between units in a system is galvanic isolation.

Typically, transformers and optical isolators are using for galvanic isolation. Analog Devices has developed a technology for the production of devices called iCoupler, which consists in packing inductive elements in conventional microcircuit housings and transferring data through a magnetic field inside the housing [10]. 
In the proposed power meter circuit (Figure 3), a digital isolator from Analog Devices ADuM4160 is used as galvanic isolation of signal lines for USB 2.0 full-speed [11] and Aimtec DC/DC module with galvanic isolation is used for power lines.

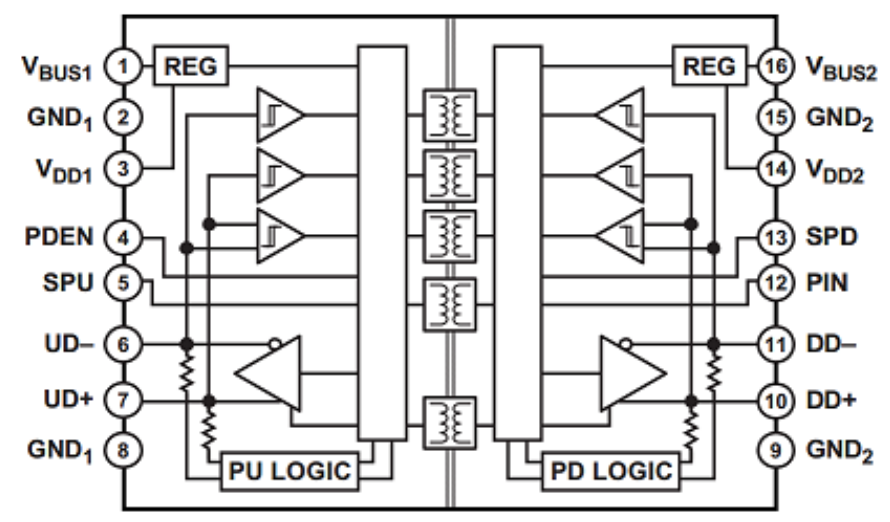

Fig. 10. Functional block diagram of galvanic isolation ADUM4160.

\section{Conclusion}

In RF measurements using circuits based on two RMS power detectors and asymmetrical power divider with attenuation $3 \mathrm{~dB}$ and $16 \mathrm{~dB}$ to different detection stages increases diode power meter dynamic range up to $90 \mathrm{~dB}$.

The example of real application such as USB power meter PLS06 with frequency range from $10 \mathrm{MHz}$ to $6 \mathrm{GHz}$ and dynamic range $70 \mathrm{~dB}$ is presented.

Also we introduced and compared the results of measuring pulse /digital modulated signals from PLS06 and other RMS power meters.

The one of the method of galvanic isolation USB interface based on special IC from Analog Devices and Aimtec for signal and power lines is described.

\section{References}

1. M.I. Bilko, A.K. Tomashevsky, P.P. Sharov, Measurement of microwave power (M .: "Soviet Radio", 1976)

2. O.S. Milovanov, N.P. Sobenin, Technique of superhigh frequencies, Textbook for high schools (Atomizdat, 1980)

3. https://www.micran.ru/productions/instrumentation/usb/pls06/

4. Agilent Fundamentals of RF and Microwave Power Measurements, Application Note 1449-2 (2001)

5. N.N. Voronin, A.S. Zagorodny, Method for expanding the area of quadratic detection of diode detectors through the use of several detector branches, UDC: 621.3.083.1

6. Patent US 2009/0315534 A1: Dec. 24, Method for eliminating the need to zero and calibrating, Richard R. Hawkins, Jon R. Sigler (2009)

7. Patent US 7830134 B2: Nov. 9, Power meter with means to eliminate the need to zero and calibrating, Richard R. Hawkins, Jon R. Sigler (2010)

8. A.S. Zagorodny, Power meters for microwave and EHF signals based on diode detectors, dis. ... cand. Those Sciences: 05.12.07, Zagorodnii Andrey Sergeevich, Tomsk (2014) 
9. TruePwr Detector $67 \mathrm{~dB}, 10 \mathrm{MHz}$ to $10 \mathrm{GHz}, \mathrm{ADL5906}$, Data Sheet

10. O. Romanov High-speed digital isolators from Analog Devices - a worthy alternative to optocouplers, Components and technologies №7 (2003)

11. Full / Low Speed 5 kV USB Digital Isolator, ADuM4160, Data Sheet 